\title{
Estimation of the number of motor units based on
} macro-EMG

\author{
P DE KONING, ${ }^{*}$ G H WIENEKE, $\dagger$ D VAN DER MOST VAN SPIJK, $\dagger$ \\ A C VAN HUFFELEN, $\dagger$ W H GISPEN,* F G I JENNEKENS
}

From the Division of Molecular Neurobiology, ${ }^{*}$ Rudolf Magnus Institute for Pharmacology and Institute of

Molecular Biology and Medical Biotechnology, University of Utrecht; Department of Clinical

Neurophysiology; $†$ and Laboratory for Neuromuscular Diseases, $\ddagger$ University Hospital, Utrecht, The

Netherlands

SUMMARY The technique of the macro-EMG was used to estimate the number of motor units in the tibialis anterior muscles of healthy subjects in a wide range of ages, and of patients with myasthenia gravis and patients with amyotrophic lateral sclerosis or spinal muscular atrophy. The results obtained suggest a decrease in the number of motor units in the tibialis anterior muscle with increasing age in normal subjects. In myasthenic patients the motor unit count was within the normal range for their age group. Patients with motor neuron disorders on the average had a very low number of motor units.

Conventional electromyographic (EMG) investigations help to differentiate between neuropathic and myopathic processes. However, they yield little quantitative data on the amount of denervation and reinnervation activity in muscles affected by neuropathic processes. The introduction of single fibreEMG (SF) and macro-EMG has provided useful quantitative information on the micro- and macrophysiology of diseased muscle. ${ }^{1-4}$ An estimate of the number of functional motor units (MU) in a muscle would, however, augment our knowledge of the balance between denervation and reinnervation.

The method described by McComas to estimate the number of MUs in the human extensor digitorum brevis muscle has several disadvantages that will be listed below. ${ }^{5-8}$ The application of the method is limited to muscles from which electrical activity can be evoked without interference from other muscles; furthermore the overlying skin has to be thin and no recordings can be made from bulky muscles. These requirements arise from the use of surface recording electrodes. The use of such electrodes makes it

Address for reprint requests: Dr P de Koning, Division of Molecular Biology, University of Utrecht, Padualaan 8, 3584 CH Utrecht, The Netherlands.

Received 9 June 1987 and in revised form 25 August 1987. Accepted 29 August 1987 difficult to record small motor unit potentials (MUPs) that occur for instance in dystrophic disorders in muscle. This insensitivity might partly explain the observation by McComas that the total number of MUs in Duchenne dystrophy is reduced, which is in disagreement with the generally agreed interpretation of concentric needle investigations. Another drawback of McComas' method is the use of amplitude measurements for the recognition and quantitation of additional recruited MUs on graded stimulation of the anterior tibial nerve. Especially in neuromuscular disorders the potential maxima of MUs might not summate temporally owing to differences in their latencies, durations and shapes. Ballantyne and Hansen $^{5}$ have improved this method by using a computer-aided system for the detection and estimation of the number of MUs in human extensor digitorum brevis. They calculated the difference in potential area between successively recruited potentials ("area of the difference potential") and stored the previously evoked muscle action potentials. Any difference in latency, duration, amplitude or shape between recruited potentials influences this "area of the difference potential", which allows more reliable recognition of additional recruited MUs. Analysis of successively recruited MUs with respect to amplitude and area of their potentials gives an estimate of the relative size of MUs. ${ }^{9}$ However, the disadvantage of using the extensor digitorum brevis as a representa- 
tive of all of the body's musculature remains. Histological investigations have shown that denervation is present in extensor digitorum brevis in normal subjects of all ages. ${ }^{10}$ Both the methods of McComas ${ }^{2}$ and Ballantyne and Hansen ${ }^{1}$ rely on gradual stimulation of the muscle nerve for additional recruitment of MUs. Milner-Brown and Brown ${ }^{8}$ have provided evidence that this technique might result in an overestimation of the number of MUs due to the spontaneous changes in motor unit potential amplitudes on repeated stimulation. Furthermore, gradual stimulation resulted in recruitment of the smallest size MUs which are not representative for the average MU size. Voluntary contraction resulted in the recruitment of MUs which were on the average twice the size of those recruited on electrical stimulation of the nerve. ${ }^{11}$

The purpose of this paper is to describe a method for estimating the number of functional MUs in the uptake area of a needle electrode, the macroelectrode. The uptake area is defined as the muscle area surrounding the electrode from which muscle fibre action potentials contribute to the recorded potential. In single-fibre EMG, fibre density is a measure of the local concentration of muscle fibres belonging to one motor unit within the uptake area of a single-fibre electrode. The mean value of recordings at 20 different positions is called the fibre density. On the analogy of the fibre density the calculation of MUs in the uptake area of the intramuscular macroelectrode is called the motor unit density (MUD). Intramuscular recording overcomes the difficulties in picking up small motor unit potentials and the restricted uptake area of the macro-electrode allow recordings in muscles that are not fully isolated from surrounding muscle tissue like the tibialis anterior muscle. On the basis of reports in the literature it can be anticipated that the size of MUs recruited on voluntary contraction are more representative of the average MU size than those recruited by means of gradual stimulation. ${ }^{11}$ This method has been applied to the tibialis anterior muscle of healthy volunteers in a wide range of ages, three patients with myasthenia gravis, four patients with amyotrophic lateral sclerosis and two patients with spinal muscular atrophy.

\section{Methods}

\section{Subjects}

Thirty-six healthy volunteers of both sexes aged between 21 and 76 years were investigated. In none of the subjects was there evidence of neurological abnormality in the investigated leg. The subjects rested comfortably in the supine position on a couch. The investigated legs were not warmed but the ambient temperature was regulated at $20-24^{\circ} \mathrm{C}$.

Three patients with myasthenia gravis (MG) aged 23, 35 and 45 years were studied, they were all symptomatic and $\overline{\underline{\sigma}}$ showed increased jitter in the tibialis anterior or a decremen- $Z$ ting response to tetanisation. Two of the patients had undergone thymectomy. While two of the patients reacted well to Mestinon treatment, the third patient benefited only from prednisone: $30 \mathrm{mg}$ every $48 \mathrm{~h}$. Four patients with amy- $\overline{\widehat{c}}$ otrophic lateral sclerosis (ALS) and two with spinal mus- $\square$ cular atrophy (SMA) aged between 59 and 73 years were studied. In one patient a diagnosis of familial ALS was made. The history of symptoms was 1 to 2 years in the ALS patients and 1 and 4 years in the two SMA patients.

\section{Recording}

A $4 \mathrm{~cm}$ long, $0.55 \mathrm{~mm}$ diameter needle electrode was used for recording (Medelec SFM 37.53114). This electrode contained two recording surfaces: the macro-recording surface is the uninsulated first $15 \mathrm{~mm}$ of the shaft (the remainder of the shaft is isolated); in the middle of the bared part of the shaft a $25 \mu \mathrm{m}$ diameter platinum electrode is exposed, which is the SF electrode. This needle electrode will be referred to as the macro-electrode.

Recordings were made on two channels. One channel (filter settings: HP: $400 \mathrm{~Hz}, \mathrm{LP}: 10 \mathrm{kHz}$ ) recorded a SF-EMG signal between the platinum electrode and the uninsulated part of the macro-electrode. The other channel (filter settings: HP: $10 \mathrm{~Hz}$. LP: $5 \mathrm{kHz}$ ) recorded the signal between the uninsulated part of the macro-electrode and the tip of $\$ \omega$ monopolar needle electrode, inserted subcutaneously jus $\vec{Q}$ above the medial ankle. The SF-EMG signal was used th trigger a digital averager to which the signal recorded on the uninsulated part of the macro-electrode was fed. Each time $\rightarrow$ the SF-signal triggered the averager, $65 \mathrm{~ms}$ of the signal $>$ recorded on the uninsulated part of the macro-electrode wक्乛 음 collected for averaging. Averaging was performed on line $\frac{\rho}{\supset}$ usually for 130-260 impulses. The SF-trigger was at $30 \mathrm{~m}$. $\vec{c}$ within the epoch of $65 \mathrm{~ms}$. The potential resulting from this averaging procedure is called the macro-motor unit potential (macro-MUP). From this macro-MUP the maximal peak-to-peak amplitude and the area under the signal during the middle $55 \mathrm{~ms}$ of the epoch of $65 \mathrm{~ms}$ were calculated. This latter calculation was made by averaging the first and last $5 \mathrm{~ms}$ of the $65 \mathrm{~ms}$ epoch and using this as a relative zero voltage line. The area enclosed by the signal and this zero voltage line was then calculated. A macro-MUP represents the temporal and spatial summation of muscle fibre action potentials belonging to one MU.

In a healthy volunteer, the endplate zone of the tibialis anterior muscle was mapped out by means of stimulation of the deep peroneal nerve and simultaneously recording the muscle action potential with a small surface electrode. The endplate zone was located at one third of the distance between the lower border of the patella and the upper border of the ankle, with the leg in a straight position. In all recordings the macro-electrode was inserted $3 \mathrm{~cm}$ distal to the endplate zone. ${ }^{12}$

For recording a supramaximal evoked muscle action potential (macro-SEMAP) the deep peroneal nerve was stimulated supramaximally near the head of the fibula by means of monopolar needle electrodes. Electrical stimuli $N$ were delivered by a constant current stimulator and consisted of rectangular pulses of $0.2 \mathrm{~ms}$ duration. The supramaximal evoked muscle action potential (SEMAP) was $\omega$ 
recorded on the macro-electrode with the electrode in the middle of an insertion channel. A ground electrode was placed between stimulation and recording electrodes.

\section{Calculation of motor unit density}

The recording procedure on the basis of which the MUD is calculated, will be outlined below. On one insertion of the macro-electrode in the tibialis anterior, five macro-MUPs were recorded at different depth during slight to moderate voluntary contraction. Contraction strength was controlled by applying counter-pressure to the foot. The five macroMUPs were taken as a representative for the collection of MUs in the uptake area of the macro-electrode. The mean amplitude and area of the five macro-MUPs was calculated. Subsequently, with the macro-electrode in the same insertion channel, the deep peroneal nerve was stimulated supramaximally and the macro-SEMAP was recorded on the macro-electrode. The number of MUs in each insertion channel was calculated from the formula:

$$
\begin{aligned}
& \text { amplitude macro-SEMAP/mean amplitude macro-MUP } \\
& \text { and }
\end{aligned}
$$
area macro-SEMAP/mean area macro-MUP

Amplitude and area macro-SEMAP is the amplitude and area of the muscle action potential recorded on the macroelectrode due to supramaximal stimulation of the deep peroneal nerve. Mean amplitude and area macro-MUP is the mean amplitude and area of the five macro-MUPS recorded in the same insertion channel on voluntary contraction. This procedure was applied at four different insertions which have about $5 \mathrm{~mm}$ spacing and are situated on a line parallel to the endplate zone of the tibialis anterior. The MUD for the muscle is the mean MUD of the four insertions. A schematic presentation is given in fig 1 .

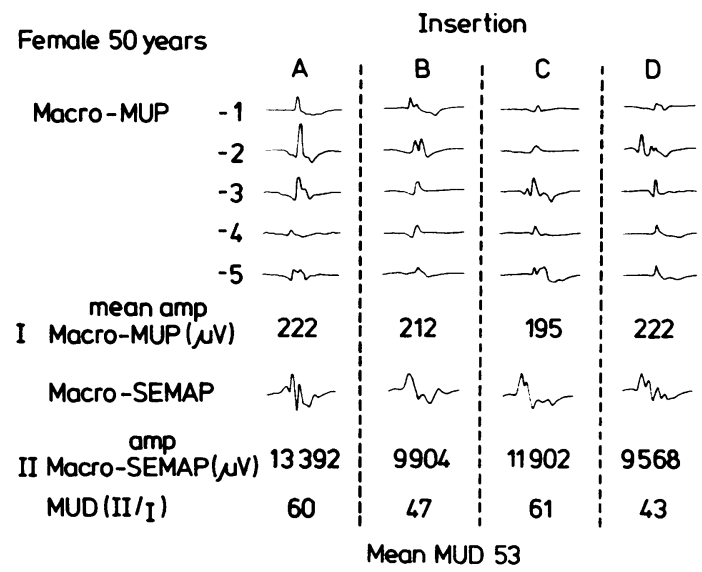

Fig 1 Schematic presentation of the recording procedure used to calculate the mean motor unit density (MUD) of a muscle.
Results

\section{Age-related changes}

The MUD based on amplitude measurements ranged between 10 and 78 while the MUD based on area measurements ranged between 14 and 95 . The relationship between the MUD and age in the tibialis anterior muscle of 36 healthy subjects is shown in fig $2 \mathrm{~A}$ and $\mathrm{D}$. In general a weak decrease of the value of the MUD with age was found, the Spearman correlations between the MUD and age were -0.48 $(p<0.01)$ for the measurements based on the amplitude and $-0.32(p<0.03)$ for the measurements based on area. The decrease of MUD with age was particularly pronounced in older persons (over 60 years). Table 1 shows the mean MUD and standard deviation for successive age groups of 20 years. In fig $2 \mathrm{~A}$ and $2 \mathrm{D}$ regression lines indicating the decrease in MUD with age are drawn (respectively $\mathrm{Y}=-0.48 \mathrm{X}$ +58 and $\mathrm{Y}=-0.36 \mathrm{X}+56)$. The mean amplitude of the macro-MUPs showed a small but significant increase with age (fig 2B; Spearman correlation = $-0.53, p<0.001$ ) whereas the mean amplitude of the supramaximal evoked muscle action potential (SEMAP) showed no tendency to decrease with age (fig 2C: Spearman correlation $=-0 \cdot 14, \mathrm{p}<0 \cdot 2$ ). The same relations are found for the area measurements, the mean area of the macro-MUP increased with age (fig $2 \mathrm{E}$; Spearman correlation = $-0.38, p<0.01)$ and again the mean area of the SEMAP showed no tendency to decrease with age (fig $2 \mathrm{~F}$; Spearman correlation $=-0 \cdot 23, \mathrm{p}<0 \cdot 1)$. The distribution of the MUD of males and females clearly showed that there was no correlation between MUD and sex. Care was taken to obtain recordings equally representing all depths. No difference in size of superficial and deep located MUs was observed. (Students $t$ test: $\mathrm{t}=0.0097, \mathrm{p}<0.48$ ).

\section{Validity of the method}

During recordings the subjects were asked to contract their tibialis anterior muscle slightly to moderately. In order to assess to what extent this recording procedure selected primarily small sized MUs, two different recording procedures were compared. In the first procedure $(\mathrm{A})$, which is normally used during MUD calculations, macro-MUPs were recorded at slight to moderate contraction. While the subject maintained a continuous contraction, the macroelectrode was carefully positioned until a SF action potential was recorded. Following procedure $A$ the macro-electrode was retracted a few millimeters and procedure B was applied. In this second procedure (B) the subject was asked to increase contraction strength until a SF action potential was recorded with the macro-electrode in that position. This latter pro- 
Amplitude
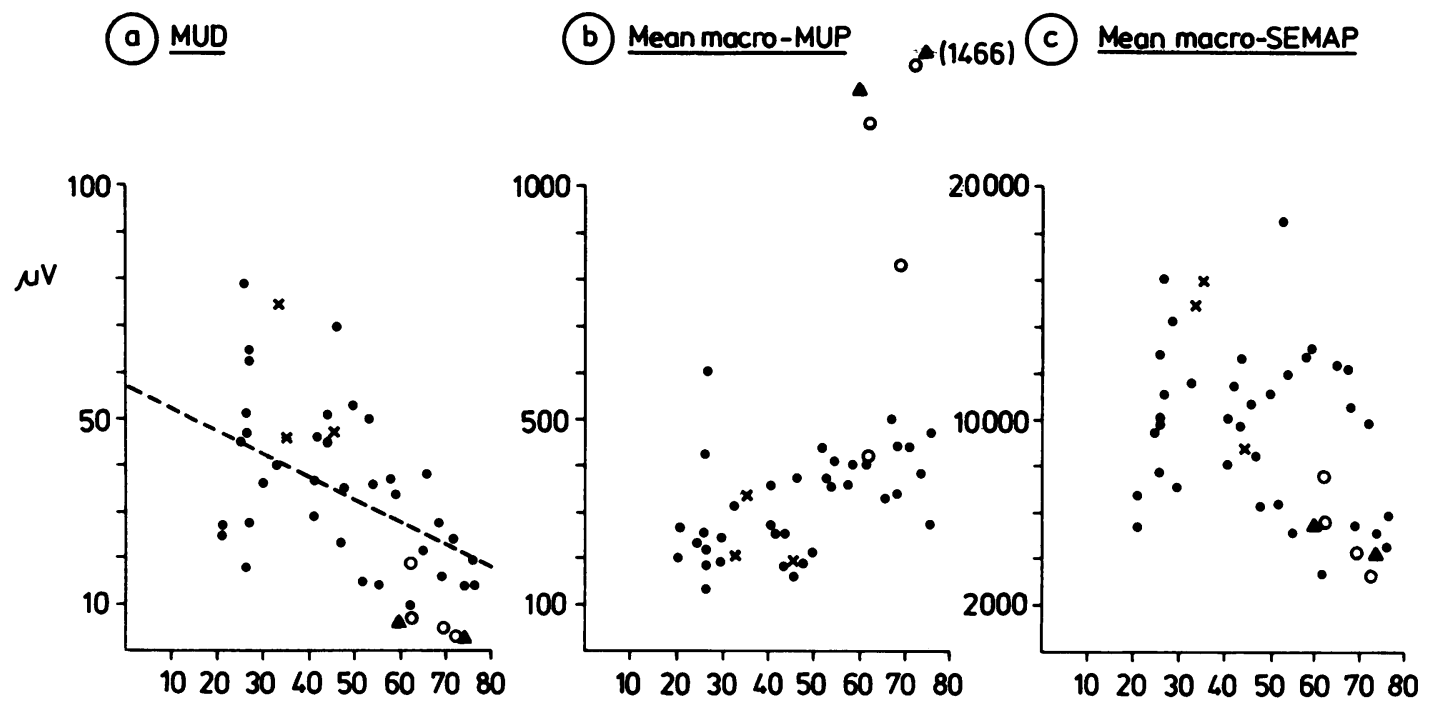

\section{Area}

(d) MUD

(e) Meanmacro-MUP

(f) Mean macro-SEMAP $\triangle(5333)$
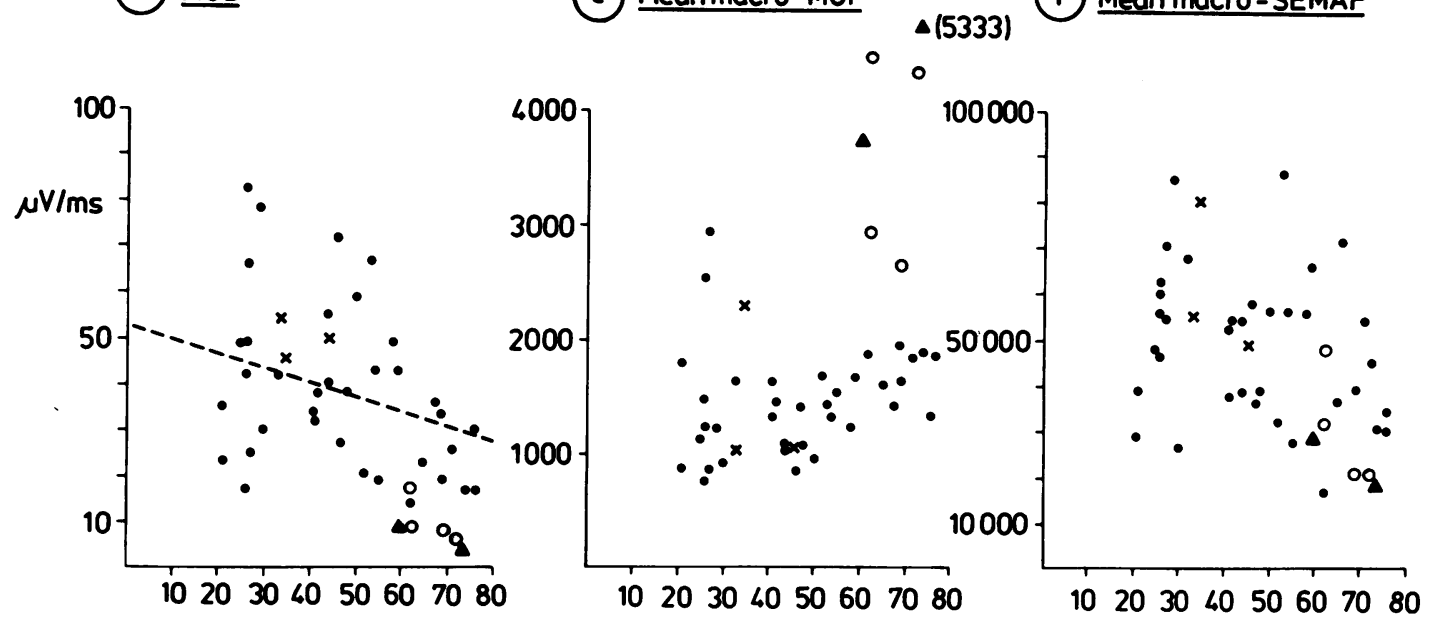

Age

Fig 2 (a) Motor unit density values, based on amplitude measurements, of the tibialis anterior muscles of 31 healthy subjects ( () , three patients with myasthenia gravis $(*)$, four patients with amyotrophic lateral sclerosis $(O)$ and two patients with spinal muscular atrophy $(\Delta)$. (b) For each subject of $A$ the mean amplitude of all recorded macro-MUPs is indicated. (c) Mean amplitude of the supramaximal evoked muscle action potential (SEMAP) of the tibialis anterior muscle of the subjects of $A$, as recorded with a macro-electrode. (d) Motor unit density, based on area measurements, of the tibialis anterior muscles of the same subjects as in a. (e). For each subject of $A$ the mean area of all recorded macro-MUPs is indicated.

(f) Mean area of the supramaximal evoked muscle action potential (SEMAP) of the tibialis anterior muscle of the subjects of $a$, as recorded with a macro-electrode. 
Table 1 Motor unit density based on amplitude or area measurements for successive age groups of 20 years.

\begin{tabular}{lll}
\hline Age $(y r)(n)$ & Amplitude & Area \\
\hline $21-40(12)$ & $43 \pm 17$ & $45 \pm 20$ \\
$41-60(15)$ & $38 \pm 14$ & $42 \pm 15$ \\
$61-80(9)$ & $21 \pm 9$ & $26 \pm 10$ \\
\hline
\end{tabular}

Indicated is the mean MUD and one standard deviation for each age group. Number of subjects per age group between brackets.

cedure sometimes necessitated considerable contraction strengths. The results of the application of these two procedures in 10 healthy subjects (five young and five older) are shown in fig 3 . In one of the younger persons (subject 10) and in three of the older persons (subjects 1,3 and 5) more larger macroMUPs were recruited using procedure B than using procedure $\mathbf{A}$. This difference reached significance only for subject 3 (Mann-Whitney U-test: $U=21, p<$ 0.05 ). Otherwise, with both procedures, higher contraction strengths resulted in the recruitment of larger macro-MUPs.

Temporal dispersion of the MU potentials which are summed in the SEMAP may result in an underestimation of the MUD based on amplitude measurements. An attempt was made to assess the possible occurrence of temporal dispersion by comparing the mean duration of the macro-SEMAP and the maximum duration of the macro-MUPs (estimated by the mean value plus 2 times the standard deviation). However, the duration can only be measured roughly owing to the extended shape of the macro-MUP and the background noise. The ratio of the SEMAP duration and maximum macro-MUP duration was determined for 10 subjects (table 2 ). The mean ratio appeared to be about 1 but there was a tendency of this ratio to become larger in the olderaged. It may be expected that if temporal dispersion is important, it should influence the MUD which is based on amplitude measurements to a greater extent than the MUD which is based on area measurements. To assess this possibility the ratio: MUD based on area measurements/MUD based on amplitude measurements was determined (fig 4). As can be seen, over the age of 45 years calculation of the MUD on the basis of area measurements resulted in a slightly higher value than the calculation based on amplitude measurements (mean ratio below 45 yr: 0.98 ; over 45 yr: $1 \cdot 27$, median test and chi square: $\chi^{2}=14 \cdot 39$, $\mathrm{p}<0.001)$.

Upon the assumption that the fibre distribution within MUs and the distribution of MUs within a muscle is homogenous, the variance of the MUD calculation between different insertions in one subject gives an estimate of the accuracy of the method. The
Sampling procedures

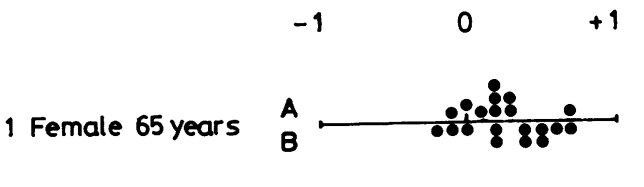

2 Male 64 years

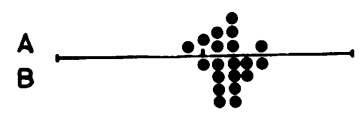

3 Male 59 years

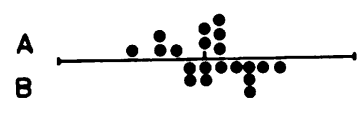

4 Female 58 years

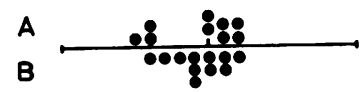

5 Male 44 years

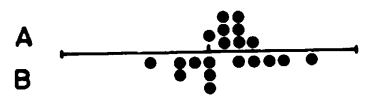

6 Female 30 years

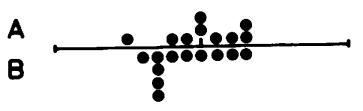

7 Female 27 years

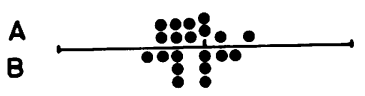

8 Male 27 years

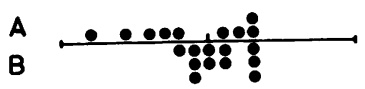

9 Female 26 years

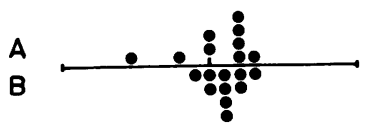

10 Male 21 years

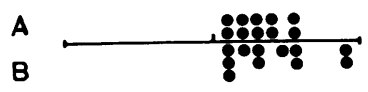

Fig 3 Amplitudes of macro-MUPs recorded from $M U$ s that were selected using two different procedures, $A$ and $B$ (see text). Each dot represents an individual $M U$. Values are given as the logarithm of the ratio of the recorded amplitude/normal median amplitude for age.

standard deviation of the calculation of the mean MUD in one subject (within variance) appeared to be about $25 \%$. Therefore, an estimate of the relative standard error of the MUD of a muscle (four insertions) will be about $12 \%$. 
Table 2 Ratio: Mean duration of the supramaximal evoked muscle action potential (SEMAP)/maximum duration of individual macro-MUPs in 10 healthy subjects

\begin{tabular}{|c|c|}
\hline $\begin{array}{ll}\$ 21 \mathrm{yr} & 0.88 \\
\text { † } 21 \mathrm{yr} & 1.23 \\
\text { o } 26 \mathrm{yr} & 0.78 \\
\text { \$ } 28 \mathrm{yr} & 0.86 \\
\text { \$ } 28 \mathrm{yr} & 1.30 \\
\text { Mean } 0.97 \pm 0.28\end{array}$ & $\begin{array}{lr}\text { o } 55 \mathrm{yr} & 1.31 \\
\text { \& } 58 \mathrm{yr} & 1.09 \\
\text { of } 59 \mathrm{yr} & 0.93 \\
\text { of } 76 \mathrm{yr} & 0.93 \\
\text { o } 76 \mathrm{yr} & 1.44 \\
\text { Mean } 1 \cdot 14 \pm 0.26\end{array}$ \\
\hline
\end{tabular}

The mean and one standard deviation of these ratios is given for both the young and old-aged group of subjects.

The reproducibility of the method was evaluated by investigating six persons on two different days. The interval between the two investigations varied between 1 week and 6 months. The difference between the results of the two MUD calculations was expressed as a percentage of the mean of these two results. With respect to MUD calculations on the basis of amplitude measurements this difference amounted to a mean of $19 \cdot 4 \%$, for MUD calculations on the basis of area measurements this difference was $9 \cdot 8 \%$.

\section{Patients}

The results of the MUD estimate in the three patients with $\mathrm{MG}$ are indicated with an asterisk (*) in fig 2 . As can be seen the MUD was within the range for subjects in their age group. Jitter was slightly to moderately increased, fibre density and the median amplitude of the macro-MUP were within the normal limits for age.

The results of the MUD calculations in the four patients with ALS $(O)$ and the two patients with SMA $(\Delta)$ are also incorporated in fig 2 . All patients except the one with familial ALS had a very low MUD for their age. The ratio of the MUD based on area measurements and MUD based on amplitude

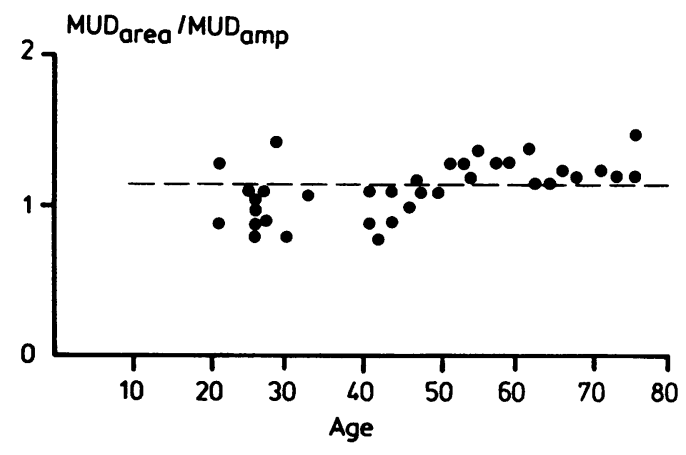

Fig 4 Each dot represents the ratio of the motor unit density (MUD) based on area measurements/MUD based on amplitude measurements, for one of the 36 healthy subjects of fig $2 A$.

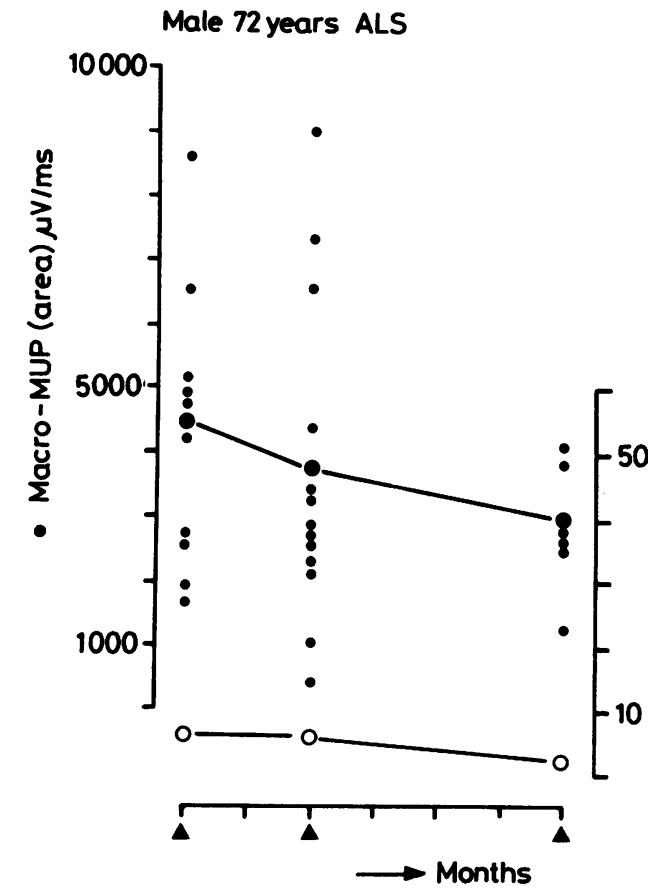

Fig 5 Repeated macro-EMG investigations over a period of 6 months in a 72 year old ALS patient. History of symptoms: 2 years. Indicated are individual $(\bullet)$ and the mean $(\bullet)$ area of the recorded macro-MUPs as well as the motor unit density, based on area measurements.

measurements was 1.44 . On repeated investigation of the patient with familial ALS, the MUD decreased. Jitter and fibre density were enhanced in all patients and the median macro-MUP amplitude exceeded the normal limit for age in all patients except the one with familial ALS. The results of repeated investigations in a 72 year old male ALS patient are shown in fig 5. While the MUD remained unchanged at two successive investigations, the mean of the macro-MUPs decreased.

\section{Discussion}

The motor unit density value represents an estimate of the number of MUs within the uptake area of a macro-electrode in a certain muscle.

It is apparent that there are two aspects in the calculation of the MUD that need to be further addressed. First, the randomness of selection of MUs from the total MU population that are used for recording a macro-MUP. The use of two different recording procedures indicated that at contraction strength normally used for recording macro-MUPs, samples are obtained predominantly from the popu- 
lation of small and medium-sized MUs. At higher contraction strengths larger MUs were recruited. In this respect it should be noted that recruitment of MUs by means of gradual nerve stimulation also resulted in the recruitment of small sized MUs. ${ }^{7811}$ The population of small and medium sized MUs is probably much larger than that of large-sized MUs (see also ref 13). However, it can be anticipated that on the basis of the recording procedure normally used the mean size of the macro-MUP will be underestimated. This results in an over-estimation of the number of MUs in the uptake area of the macroelectrode. Stallberg and Fawcett ${ }^{14}$ reported that the amplitudes of macro-MUPs of MUs recruited at $30 \%$ of maximum force can be five times those recruited below $10 \%$. In our measurements we found a considerable variation in the amplitude and area of the macro-MUPs. The amplitude and area of the supramaximal evoked muscle action potential also varied among the different insertions. These two variations are reflected in the standard deviation of the MUD for the whole muscle. It can be questioned whether this variation is only due to sampling variation or whether it also reflects a biological variation in the distribution of MUs within a muscle.

Retrospectively, it became apparent that the young aged subjects with a low MUD were very active sportsmen. Their low MUD was caused by the fact that their mean macro-MUP values were rather high (slightly exceeding the maximum reference values) whereas their macro-SEMAP although in the higher range did not deviate from the mean population. This combination of findings might have obscured their MUD calculations, at the same time disturbing the overall regression analysis of MUD versus age. If the three subjects should have been excluded from the regression analysis, the Spearman correlation between MUD based on amplitude measurements and age would have increased from -0.48 to -0.63 whereas the Spearman correlation between MUD based on area measurements and age would have increased from -0.32 to -0.50 .

The second aspect that needs to be elaborated is the selection of either amplitude or area measurements for calculation of the MUD. In general, temporal dispersion between MUs that contribute to the SEMAP will result in lower MUDs. It can be expected that amplitude measurements will be more influenced by temporal dispersion than area measurements. Although comparison of the duration of macroMUPs and macro-SEMAP did not suggest a large temporal dispersion, for subjects aged over 45 years MUD which is based on area measurements appeared to be slightly but significantly larger than MUD which is based on amplitude measurements. It is possible that the temporal dispersion among MUs increases with age. Another possible explanation for the fact that the difference became apparent only over 45 years of age, may be the fact that younger subjects had generally smaller MUs. With smaller MUs, area measurements are more influenced by the background noise and therefore the mean area of the macro-MUP will be calculated erroneously high, resulting in an underestimation of the MUD. Therefore we suggest that amplitude measurements for MUD calculations be used below 45 years of age and that area measurements for MUD calculations be used over 45 years of age in healthy persons. Patients with neuromuscular disorders can be expected to have more temporal dispersion of their motor unit potentials than healthy persons. It is probably best to calculate their MUD values on the basis of area measurements. Another point which favours the use of area measurements in the calculation of the MUD is the fact that the calculation of the MUD on the basis of area measurements showed less variation on repeated investigation of the same subject.

The MUD estimates the number of MUs in the restricted uptake area of the macro-electrode. Although it is difficult to assess the exact size of this uptake area, it is probably in the order of $6-8 \mathrm{~mm}$ (diameter). The uptake area of an EMG-electrode is the muscle area surrounding the electrode from which muscle fibre action potentials contribute to the recorded potential. In general the contribution of a muscle fibre decreases rapidly with increasing distance from the electrode. The border of the uptake area is usually defined as the distance at which the amplitude of a muscle fibre action potential has decreased to $5 \%$ of its maximum (a muscle fibre action potential is recorded with maximum amplitude when the muscle fibre lies against the electrode). In this paper two different uptake areas have to be distinguished. First, the muscle fibre uptake area of the macro-electrode, that is the area from which muscle fibre action potentials belonging to one MU contribute to a macro-MUP. Based on data reported by Stålberg (ref 4, fig 4) the muscle fibre uptake area is estimated to have a radius of $2 \mathrm{~mm}(\mathrm{R})$. Therefore the macro-MUP of a MU is constituted from those muscle fibres of that MU, which lie in a cylinder with a radius of about $2 \mathrm{~mm}$ and a length of about $19 \mathrm{~mm}$ (uninsulated part of the shaft $(15 \mathrm{~mm}$ ) plus $2 \times 2 \mathrm{~mm}$ ) surrounding the macro-electrode. Second, the MU uptake area of the macro-electrode, that is the area from which MUs contribute to a macro-SEMAP. This latter uptake area is directly dependent on the radius of a MU territory (D). For as long as one muscle fibre belonging to a certain MU lies within the muscle fibre uptake area of the macro-electrode, this MU contributes to the macro-SEMAP. The MU uptake area of the macro-electrode therefore has a 
radius of about $D+R$. On the analogy of a muscle fibre, the contribution of a MU to the macro-SEMAP decreases rapidly to zero when the distance from the macro-electrode to the centre of a MU increases from D-R to D + R. The radius of the MU uptake area of the macro-electrode will therefore be by approximation D. The mean cross-section of MU territories in the tibialis anterior muscle is about $8 \mathrm{~mm} .{ }^{15}{ }^{16}$ The MU uptake area of the macro-electrode in the tibialis anterior muscle will therefore be a cylinder with a radius of about $4 \mathrm{~mm}$ and a length of about $23 \mathrm{~mm}$. In line with this suggestion is the fact that the MUD ranges between 82 and 10 , whereas estimates of the total number of MUs within the tibialis anterior muscle based on axon counts give a number of 370 $\mathrm{MUs}^{7}$ and 455. ${ }^{17}$

Estimation of the MUD is not restricted to a muscle that is fully isolated from surrounding muscles like the extensor digitorum brevis muscle. The recording of muscle activity is triggered by an intramuscular action potential generated by the muscle under investigation. The uptake area of the macro-electrode is confined to about $8 \times 23 \mathrm{~mm}$. Therefore on stimulating the nerve innervating the muscle under investigation, for example, the deep peroneal nerve, activity will be predominantly recorded from muscle fibres nearby the macro-electrode and the contribution from other muscles innervated by the same nerve will be small. The tibialis anterior muscle seems more appropriate than the extensor digitorum brevis to serve as a representative of all of the body's musculature ${ }^{1018}$ It was usually not difficult to record from MUs for 40-60 s at sustained contraction. Hanner $\mathrm{z}^{19}$ observed that MUs recruited on voluntary contraction below $30 \%$ of the maximum tonic tension of the tibialis anterior muscle discharged continuously. At these contraction strengths recordings will be made predominantly from slow type MUs which are made up from type I muscle fibres. It has been reported by Johnson et $a l^{20}$ that $73 \%$ of the muscle fibres in the tibialis anterior muscle are type I and $27 \%$ type II. The fibre types lie intermingled and there is no significant difference in the distribution of type I and type II fibres at different depths within the muscle. In the present study no differences in size were found between superficial and deep located MUs. The macro-MUP is, amongst others, dependent on the muscle fibre diameter and spatial and temporal dispersion of muscle fibres action potentials. Since the macro-SEMAP is also dependent on the muscle fibre diameter, though to a lesser degree, the division should result in a reduced variation in MUD. We calculated the variation of the MUD in the younger persons and found it to be less than the variation which could be expected if the variation of macro-SEMAP and macro-MUP were independent. This implies that taking the macro-SEMAP in the nominator of the MUD calculations reduces the interindividual variation due to the differences in the morphology of muscles, for example, muscle fibre diameter.

The results of this method as applied to the tibialis anterior muscle of healthy subjects nonetheless show that there is a considerable scatter in MUD values. The differences in MUD values between subjects were much larger than the standard error of the MUD value of individual subjects which makes us believe that the inter individual variation in MUD is true.

The MUD decreased with age, which may indicate that there is a loss of MUs with increasing age. This is in line with the reports by McComas et al, ${ }^{7}$ Campbell et $a l^{21}$ and Hansen and Ballantyne ${ }^{5}$ who estimated the total number of MUs within the extensor digitorum brevis muscle and Brown ${ }^{22}$ who estimated the total number of MUs in thenar muscles. In agreement with these authors we also found that, despite a considerable reduction in the number of MUs in the older persons, strength was maintained. Death of anterior horn cells with age interferes with a process where individual MUs are probably continuously losing and gaining small numbers of muscle fibres within their own territories and within the overlapping territories of other units. These two processes result in a continuous reshuffling of MUs. Histologically, these processes manifest themselves as a reduction in the number of muscle fibres, ${ }^{23}$ an increased occurrence of fibre type grouping ${ }^{24}$ and a reduction of the number of anterior horn cells. ${ }^{25}$ On electrophysiological investigation aging of the neuromuscular system expresses itself, among other ways, in SF-EMG as an increase in fibre density. In macro-EMG, as becomes also apparent in the present study, the mean amplitude and area of the macro-MUP increase (see also ref 14). The fact that the macro-SEMAP just failed to decrease significantly with age although there was a marked decrease in macro-SEMAP over the age of 60 years, is probably caused by the large variation in macro-SEMAP under the age of 60.

In the myasthenic patients we have examined, the MUD in the tibialis anterior muscle was not significantly different from that in control subjects. The amplitudes of the macro-MUPs were within the normal limits for age. This does not indicate that degeneration was absent in these patients. In fact, in two of the myasthenic patients, concomitant blocking and jitter had been observed on previous SF investigations, suggesting degeneration and regeneration. ${ }^{1}$ This degeneration is apparently not accompanied by loss of motor neurons, neither is there an increase in MU size which can be picked up with the macro-EMG. The results are in line with the reports by Ballantyne and Hansen ${ }^{59}$ which indicate a normal MU count in the EDB muscle as well as a normal 
amplitude and area of the recruited MUs in myasthenic patients. The MUD in the tibialis anterior muscle of patients with motor neuron disorders we have investigated thus far, is very low except for one patient with familial ALS. In all but this latter patient the mean area of the macro-MUP was increased to between two and five times the mean value of control subjects of the same age. Hansen and Ballantyne ${ }^{26}$ argue that once the disease process affects an anterior horn cell, this cell deteriorates very rapidly. However, we believe that this process of deterioration of the motor unit is a more slowly progressing process. On repeated investigation of the same patient with ALS, the MUD in the tibialis anterior muscle remained unchanged, whereas the mean area of the macroMUPs decreased, indicating that individual MUs became smaller. Another explanation for this observation is that there was a predominant loss of large MUs, which resulted in a packing of small-size MUs. We cannot differentiate definitely between these two possibilities. However, the presented results as well as other reports ${ }^{27}$ favour the idea that the deterioration of individual MUs progresses slowly.

In conclusion, determination of the motor unit density in a muscle can give additional quantitative information on the balance between degeneration and regeneration in disorders of the peripheral nervous system. The fact that this method can easily be combined with single-fibre and macro-EMG makes it very useful, in our opinion.

\section{References}

1 Stålberg E, Trontelj JV. Single Fibre Electromyography. Woking, England: Mirvalle Press, 1979.

2 Stålberg E. Macro EMG, a new recording technique. $J$ Neurol Neurosurg Psychiatry 1980;43:475-82.

3 Stålberg E. Macroelectromyography in reinnervation. Muscle and Nerve 1982;5:135-8.

4 Stålberg E. Macro-EMG. Muscle and Nerve 1983;6:619-30.

5 Ballantyne JP, Hansen S. A new method for the estimation of the number of motor units in a muscle. J Neurol Neurosurg Psychiatry 1974;37:907-15.

6 De Weerd J. Quantification methods in electromyography and electroneurography. In: Notermans SLH, ed. Current Practice of Clinical Electromyography. Amsterdam: Elsevier Science Publishers 1984:479-521.

7 McComas AJ, Fawcett PRW, Campbell MJ, Sica REP. Electrophysiological estimation of the number of motor units within a human muscle. J Neurol Neurosurg Psychiatry 1971;34:121-31.

8 Milner-Brown HS, Brown WF. New methods of estimating the number of motor units in a muscle. J Neurol Neurosurg Psychiatry 1976;39:258-65.

9 Ballantyne JP, Hansen S. Computer method for the analysis of evoked motor unit potentials. Control subjects and patients with myasthenia gravis. $J$ Neurol Neurosurg Psychiatry 1974;37:1187-94.

10 Jennekens FGI, Tomlinson BE, Walton JN. The extensor digitorum brevis: histological and histochemical aspects. J Neurol Neurosurg Psychiatry 1972; 35:124-32.

11 Kadrie HA, Ytes SK, Milner-Brown HS, Brown WF. Multiple point electrical stimulation of ulnar and median nerves. J Neurol Neurosurg Psychiatry 1976;39:973-85.

12 Nandedkar S, Stålberg E. Simulation of Macro-EMG motor unit potentials. Electroencephalogr Clin Neurophysiol 1983;56:52-62.

13 McPhedran AM, Wuerker RB, Henneman E. Properties of motor units in a homogeneous red muscle (soleus) of the cat. J Neurophysiol 1965;28:71-84.

14 Stålberg E, Fawcett PRW. Macro-EMG in healthy subjects of different ages. J Neurol Neurosurg Psychiatry 1982;45:870-8.

15 Erminio F, Buchtal F, Rosenfalck P. Motor unit territory and muscle fiber concentration in paresis due to peripheral nerve injury and anterior horn cell involvement. Neurology 1959;9:657-71.

16 Hilton-Brown P, Stålberg E. Motor unit size in muscular dystrophy, a macro-EMG and scanning-EMG study. J Neurol Neurosurg Psychiatry 1983;46:996-1005.

17 Enoka RM, Stuart DG. Henneman's "size principle": current issues. TINS 1984;75:226-8.

18 Roselle N, Stevens A. Unexpected incidence of neurogenic atrophy of the extensor digitorum brevis muscle in young normal adults. In: Desmedt JE, ed. New Developments in Electromyography and Clinical Neurophysiology. Basel: Karger, 1973:69-70.

19 Hannerz J. Discharge properties of motor units in relation to recruitment order in voluntary contraction. Acta Physiol Scand 1974;91:374-84.

20 Johnson MA, Polgar J, Weightman D, Appleton D. Data on the distribution of fiber types in thirty six human muscles. An autopsy study. J Neurol Sci 1973;18:111-29.

21 Campbell MJ, McComas AJ, Petito F. Physiological changes in ageing muscles. J Neurol Neurosurg Psychiatry 1973;36:174-82.

22 Brown WF. A method for estimating the number of motor units in thenar muscles and the changes in motor units count with aging. I Neurol Neurosurg Psychiatry 1972;35:845-52.

23 Lexell J, Downham D, Sjöström M. Distribution of different fiber types in human skeletal muscles. $J$ Neurol Sci 1986;72:211-22.

24 Jennekens FGI, Tomlinson BE, Walton JN. Histochemical aspects of five limb muscles in old age. J Neurol Sci 1971;14:259-76.

25 Tomlinson BE, Irving D. The number of limb motor neurons in the human lumbosacral cord throughout life. J Neurol Sci 1977;34:213-9.

26 Hansen S, Ballantyne JP. A quantitative electrophysiological study of motor neurone disease. J Neurol Neurosurg Psychiatry 1978;41:773-83.

27 Stålberg E. Electrophysiological studies of reinnervation in ALS. In: Rowland LP, ed. Human Motor Neuron Diseases. New York: Raven Press, 1982:47-59. 